\title{
Emergence of orientation selective simple cells simulated in deterministic and stochastic neural networks
}

\author{
M. Stetter, E. W. Lang, A. Müller \\ Institut für Biophysik und physikalische Biochemie, Universität Regensburg, POB 1010 42, W-8400 Regensburg, \\ Germany
}

Received: 4 July 1992/Accepted in revised form: 6 November 1992

\begin{abstract}
The processing of visual data in area 17 of the mammalian cortex is mainly performed by cells with receptive fields which are tuned to different orientations of input stimuli. The mechanisms underlying the emergence of receptive field properties of orientation selective cells are not well understood up to now. Recently, some models for the prenatal development of the receptive fields of orientation selective simple cells have been proposed, which emerge in neural networks trained by Hebb type unsupervised learning rules. These models, however, use different network architectures and are restricted to the case of identical input neurons. In this work, a biologically motivated neural network model with a general architecture is presented. It is trained with a Hebb type updating rule and with uncorrelated input. The input neurons are identified with retinal ganglion cells and exhibit mature Mexican hat type receptive fields. If the receptive fields of the input neurons have identical properties (deterministic model), a set of parameter domains is found, which characterize different kinds of receptive field maturation behaviour of the network. Results obtained by other authors with similar models are contained in this description as special cases. In addition, the more general and rarely investigated stochastic model, where random variations of the parameters describing the receptive fields of the input neurons occur, is investigated. A high sensitivity of the network against these random variations is obtained. In case of large variations of receptive field parameters of the ganglion cells, a qualitatively new kind of maturation behaviour appears. A significant part of the synaptic connections from ganglion cells to the cortical cell is removed and small simple cell receptive fields with only few lobes emerge. The stochastic model is found to provide a better description of the size, scatter and structure of receptive fields present in biological systems, than the deterministic model.
\end{abstract}

Correspondence to: M. Stetter

\section{Introduction}

Over the past 30 years, much experimental work has been devoted to enlighten the functional architecture of the mammalian visual cortex. The cortical cells were found to have well defined response properties to external stimuli and to be arranged in independent overlapping columnar structures. (Hubel and Wiesel $1968,1977)$. One of the most striking features of cortical cells is orientation selectivity, which was first described by Hubel and Wiesel (1959) and further investigated by several authors (Hubel and Wiesel 1962, 1968, 1977; Schiller et al. 1976a, b). They describe the receptive field profiles of the great majority of orientation selective simple cells to have at most two or three lobes of alternating response to external light stimuli. Surprisingly, in macaque monkey these structures form prior to any visual experience (Wiesel and Hubel 1974).

These physiological findings have led to several theoretical investigations modelling the functional properties of orientation selective simple cells (Marčelja 1980; Daugman 1985) as well as the emergence of their receptive field profiles. Due to evidence for Hebb type synaptic plasticity in biological neural systems (Hebb 1949; for a review see Brown et al. 1990), models for the emergence of orientation selective receptive field profiles as a consequence of unsupervised Hebbian learning were introduced (Linsker 1986a, b, 1990a; Kammen and Yuille 1988; Yuille et al. 1989; for a review see Linsker 1990b).

Linsker (1986a, b) showed that stable orientation selective cells develop in a linear multilayer feed forward neural network using a Hebb type learning rule and uncorrelated white noise as input. In his model, only neurons within a finite area of one layer can have connections to a single postsynaptic neuron of the subsequent layer (finite connection density function).

Kammen and Yuille (1988) showed, that the emergence of orientation selectivity in Linsker's model can be understood to result from a spontaneous symmetry breaking within the energy function on which their learning rule performs gradient descent. This model 
uses infinite range connection density functions and therefore yields infinitely sized receptive fields.

A more recent model (Yuille et al. 1989) describes the emergence of orientation selective receptive field profiles as a result of small uniform eccentricities in the correlation function of the activities of the input neurons. Again there are no restrictions to the receptive field size and in this model a modified Oja's learning rule was used (Oja 1982) which was shown to lead to cells performing principal component analysis to their input stimuli (which is not the case for Linsker's rule).

Finally, Linsker (1990a) showed that all receptive field profiles of cortical cells which perform principal component analysis to a given input and which form under translationally invariant input correlation functions can be written as

$(n, l):=f_{n}(r) \cos (l \vartheta+\varphi)$.

This brief review shows, that previous results concerning the emergence of orientation selective receptive field profiles were obtained using quite different assumptions concerning learning rules, connection density functions and correlations of neural activities within the input layer of the model neural networks.

In this paper a systematic description of the behaviour of a simple model network is given with input neurons which have mature Mexican hat type receptive field profiles. The maturation process of synaptic couplings between ganglion cells and a cortical cell is guided by a general Hebb type learning rule extracting the principal component of the input, which is taken as uncorrelated white noise. To describe the system and its behaviour, a set of parameters characterizing correlations of neural activity within the input layer as well as quantitative measures for orientation selectivity, spatial frequency and their respective bandwidths are introduced.

The paper is organized as follows. In the first part, the case of translationally invariant correlation functions is investigated using the same set of parameters for all neurons. It is shown, how all results described above can be obtained with this model and how all these results are related. The existence of parameter domains is shown, which are not described systematically so far and which can be used to unify earlier descriptions about the emergence of orientation selective receptive fields.

In the second part of this paper, random variations of receptive field parameters of the ganglion cells (henceforth called static noise) are introduced into the model network leading to a correlation function which is no longer translationally invariant. This model is referred to as the stochastic model. It is found that static noise causes all but bilobed receptive field profiles to become unstable. While the parameter domain boundaries vanish for strong noise, a new mechanism of receptive field formation appears, which is based on synapse decoupling and which leads to a correct description of several receptive field properties found for simple cells in biological systems.
In Sect. 2, a definition of the model, the learning rule and the measures for some receptive field properties are given. In Sects. 3 and 4, the behaviour of the deterministic and the stochastic model is reported. Section 5 contains a discussion, where the results obtained are compared to physiological data and a brief summary of the results is given in Sect. 6 .

\section{The model}

\subsection{A biologically motivated network architecture}

As a starting point for the construction of a monocular model neural network a two dimensional layer consisting of $N$ retinal ganglion cells is considered, whose signal activities are sent through the lateral geniculate nucleus (LGN) to a single neuron $n$ located in layer IVc of the primary visual cortex (Fig. 1a). The following assumptions are made:

1. The LGN cells make no contribution to signal processing and are therefore neglected. This includes the feedback connections from the cortex to the LGN (which in fact might be of great importance (Mumford 1991)).

2. All temporal neuronal response properties are neglected also in the present model. In particular the responses of transient $\mathrm{Y}$-ganglion cells and a description of direction selectivity of cortical cells are beyond the scope of this paper.

3. All neurons are assumed to have a linear transfer function which is identical for all ganglion cells.

4. The ganglion cells have mature Mexican hat type receptive field profiles and are located at positions described by vectors $\mathbf{r}_{a}$. Each position is occupied by an on-center as well as an off-center ganglion cell. Both cell types exhibit excitatory connections with efficacies $w_{a}^{\text {on }}$ and $w_{a}^{\text {off }}$ to the cortical neuron. Because of the assumed linearity an off-center cell can be interpreted as an on-center cell with negative synaptic efficacy $-w_{a}^{\text {off }}$ leading to a total efficacy $w_{a}=w_{a}^{\text {on }}-w_{a}^{\text {off }}$ for the pair of ganglion cells located at $\mathbf{r}_{a}$. Note, that $w_{a}$ can change its sign which is not possible for the purely excitatory efficacies $w_{a}^{\text {off }}$.

5. The position $\mathbf{R}_{a}$ of the axonal trees of the ganglion cells in layer IVc are assumed to be in strict retinotopic order. Therefore $\mathbf{R}_{a}$ can be identified with the position $\mathbf{r}_{a}$ of the ganglion cells up to a constant magnification factor $m: \mathbf{R}_{a}=m \mathbf{r}_{a}$ (Fig. 1a).

6. The synaptic connections are homogeneously distributed over the space occupied by the afferent axonal trees (radius $\varrho_{a}$ ) as well as over the dendritic tree of the cortical neuron (radius $\varrho_{n}$ ). The probability for a synaptic connection between a ganglion cell $a$ and the cortical neuron $n$ and therefore the density $\eta\left(r_{a}\right)$ of the ganglion cells connected to $n$ is then roughly proportional to the overlap of the volumina of the axonal tree of the ganglion cell and the dendritic tree of the cortical cell (Fig. 1a). Because thalamocortical axonal terminals in mature systems are found to spread over two or more ocular dominance columns (Hubel and Wiesel 


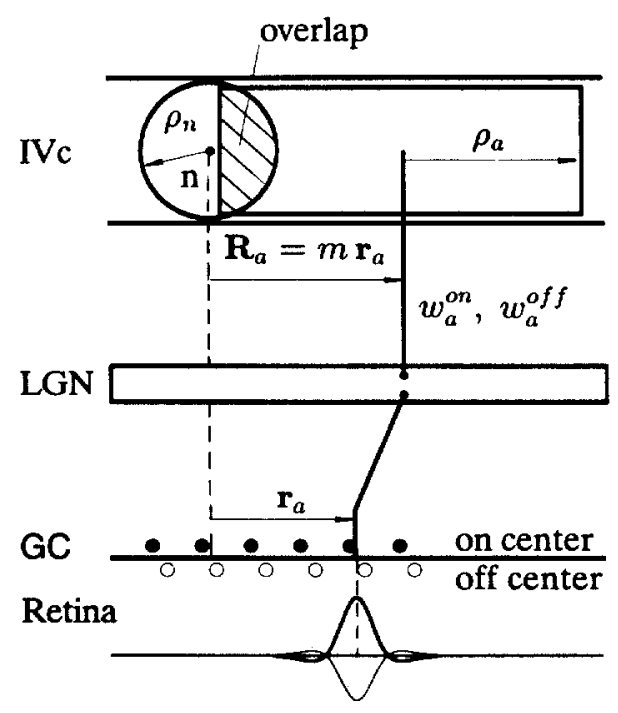

a
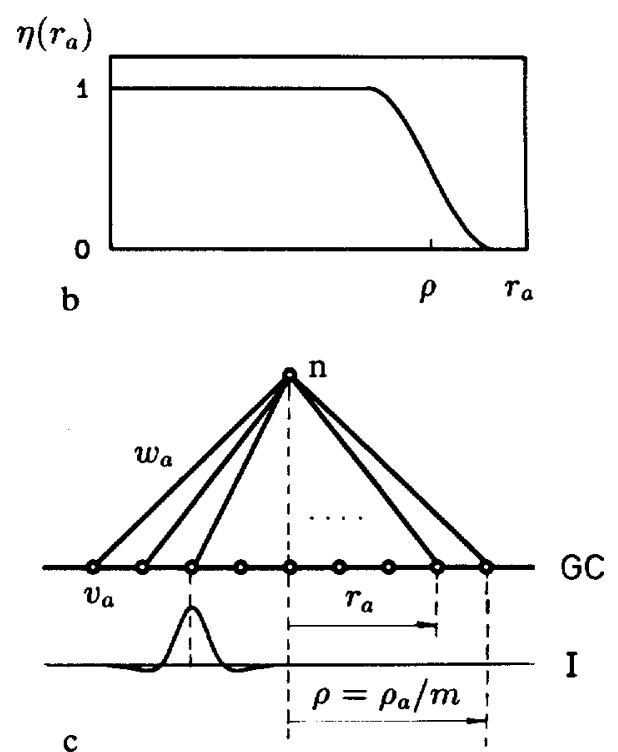

Fig. 1. a Schematic plot of the retinocortical pathway in mammals used as a starting point for the construction of the present model network. b The density of synaptic connections between ganglion cells

1977; Lund 1988), it is suggested, that in an immature system the terminals of an afferent axon are homogeneously distributed over a wide range $\left(\varrho_{a} \geqslant 0.5 \mathrm{~mm}\right)$ which is large compared to the radius of a typical stellate cell of layer IVc $\left(\varrho_{n} \approx 0.15 \mathrm{~mm}\right)$. Hence, $\varrho_{a} \gg \varrho_{n}$ holds. The connection density $\eta\left(r_{a}\right)$ for $\varrho_{n} / \varrho_{a}=0.2$ is displayed in Fig. 1b. This function can be closely approximated by a Fermi function. Even a Heavyside step function is obviously a better approximation than the generally used gaussian distribution. Therefore, a step like connection density is used for simplicity, which is defined for the ganglion cell layer as:

$\eta\left(r_{a}\right)=\left\{\begin{array}{l}1: r_{a} \leqslant \varrho \equiv \varrho_{a} / m \\ 0: r_{a}>\varrho\end{array}\right.$

Using these assumptions one arrives at a simple two layered linear neural network which corresponds to Linsker's layers $F$ and $G$ but uses a widespread step like density function (Fig. 1c). Later in this work it will be shown, that the projection radius $\varrho$ is an important parameter determining the type of the emerging receptive field. Further, a mechanism for a dynamic reduction of $\varrho$ is suggested, which leads in a natural way to receptive field profiles often observed in biological systems.

\subsection{Dynamics and learning rule}

The network described above is initialized with small random weights $w_{a}$ and then trained by presenting a set of input patterns $\pi$ where $\xi_{\pi}(\mathbf{r})$ is the photoreceptor activity at position $\mathbf{r}$ during the application of the pattern. The input patterns $\pi$ are assumed to cause uncorrelated white noise activity in the photoreceptor layer (prenatal situation) with zero ensemble average: $\left\langle\xi_{\pi}(\mathbf{r})\right\rangle_{\pi}=\mathbf{0} \quad \forall \mathbf{r}$. and the cortical target cell as a function of the ganglion cell position. c A simple model network for the situation displayed in a with input layer $I$, ganglion cell layer $G C$ and the cortical cell $n$

Taking the continuum limit in the photoreceptor layer, the activity $v_{a}$ of the ganglion cell $a$ is obtained by convolving the input pattern $\xi_{\pi}(\mathbf{r})$ with the receptive field profile $u_{a}(\mathbf{r})$ (the subscript of $u_{a}$ is necessary, because each ganglion cell $a$ might have a different receptive field profile):

$v_{a}=\int \xi_{\pi}(\mathbf{r}) u_{a}\left(\mathbf{r}-\mathbf{r}_{\mathrm{a}}\right) \mathrm{d} \mathbf{r}$.

Accordingly, the ensemble average of $v_{a}$ vanishes also: $\left\langle v_{a}\right\rangle_{\pi}=\int\left\langle\xi_{\pi}\right\rangle_{\pi} u_{a} \mathrm{~d} \mathbf{r}=0$. The activity of the cortical target neuron $s_{n} \equiv s$ is then given directly as its total weighted input

$s=\sum_{a}^{N} w_{a} v_{a}$.

The dynamic evolution of the synaptic efficacies $w_{a}$ of the ganglion cells to the target neuron $n$ is determined by a Hebb type update rule

$\delta w_{a}=\varepsilon\left(s v_{a}-\tilde{f}(\mathbf{v}, \mathbf{w}) w_{a}\right)$.

The ensemble average of this rule over all input patterns is given as

$\Delta w_{a} \equiv\left\langle\delta w_{a}\right\rangle_{\pi}=\varepsilon\left(\sum_{b} G_{a b} w_{b}-f(\mathbf{w}) w_{a}\right)$,

where $G_{a b}=\left\langle v_{a} v_{b}\right\rangle_{\pi}$ are the correlation matrix elements of the ganglion cell activities and $f(\mathbf{w})=\langle\tilde{f}(\mathbf{v}, \mathbf{w})\rangle_{\pi}$. The function $f$ is introduced to keep the weights $w_{a}$ finite and has to be chosen in a way so that rule (6) extracts the principal component of $\mathbf{G}$. Examples are given by Oja $(1982)$ as $f(\mathbf{w})=\mathbf{w}^{T} \mathbf{G w}$ and by Yuille et al. (1989) as $f(\mathbf{w})=\mathbf{w}^{T} \mathbf{w}$, where $\mathbf{w}^{T}$ is the transpose of the vector $w$. The result of a training of the network with these learning rules is an arbitrary weight vector in the subspace spanned by the eigenvectors to 
the maximal eigenvalue of the correlation matrix $\mathbf{G}$. The behaviour of this model system can therefore be analyzed by simply calculating the principal component vector (vectors) of $\mathbf{G}$ for different parameter sets which are introduced in the next subsection.

\subsection{Calculation of the correlation matrix}

The calculation of the correlation matrix elements $G_{a b}$ of the ganglion cell activities starts from the well known fact (Yuille et al. 1989), that for zero average activities the $G_{a b}$ are determined by the feed forward synaptic strengths $u_{a}$ and $u_{b}$ of the input layer and the correlation function $G\left(\mathbf{r}, \mathbf{r}^{\prime}\right)$ within this layer as

$G_{a b}=\iint u_{a}\left(\mathbf{r}-\mathbf{r}_{a}\right) G\left(\mathbf{r}, \mathbf{r}^{\prime}\right) u_{b}\left(\mathbf{r}^{\prime}-\mathbf{r}_{b}\right) \mathrm{d} \mathbf{r} \mathrm{d} \mathbf{r}^{\prime}$.

Since in the photoreceptor layer of the present model uncorrelated input is applied, $G\left(\mathbf{r}, \mathbf{r}^{\prime}\right)=\delta\left(\mathbf{r}-\mathbf{r}^{\prime}\right)$ holds yielding

$G_{a b}=\int u_{a}\left(\mathbf{r}-\mathbf{r}_{a}\right) u_{b}\left(\mathbf{r}-\mathbf{r}_{b}\right) \mathrm{d} \mathbf{r}=\int u_{a}(\mathbf{r}) u_{b}\left(\mathbf{r}-\mathbf{r}_{a b}\right) \mathrm{d} \mathbf{r}$,

where $\mathbf{r}_{a b}=\mathbf{r}_{b}-\mathbf{r}_{a}$. The receptive field profile $u(\mathbf{r})$ of a ganglion cell is written as a difference of gaussians for the receptive field center and surround and their widths are labeled $R_{c}$ and $R_{s}$ respectively (Fig. 2a). For each gaussian a small excentricity characterized through its absolute value $e \geqslant 1$ ( $e=1$ corresponds to rotational symmetry) and its angle $0 \leqslant \varphi \leqslant \pi$ with respect to a reference axis can be applied (Fig. 2b). The offset $z$ determines the strength of the surround of the receptive field. $z>0$ describes weak spatial antagonism, $z<0$

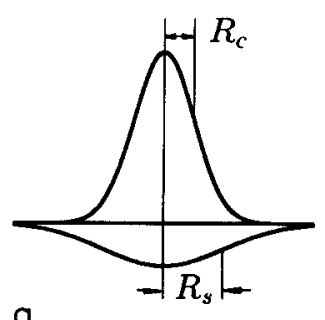

a

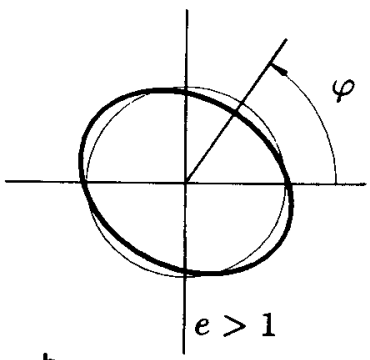

b

Fig. $2 \mathrm{a}-\mathbf{d}$. The receptive field parameters of the ganglion cells: a The radii of the receptive field center and surround $R_{c}$ and $R_{s}, b$ the magnitude $e$ and direction $\varphi$ of the eccentricity and $\mathrm{c}$ the offset $z$. $R_{0}$ represents the absolute radius of the receptive field center. d shows the two gaussians of the eccentrical gaussian function (11) for $D=0.2$ (thick lines) and the second order approximation of the wider part (thin line) leading to (12) strong antagonism of the surround (Fig. 2c) and $z=0$ leads to $\int u(\mathbf{r}) \mathrm{d} \mathbf{r}=0$ (balanced antagonism). Using these parameters, the receptive field profile of a ganglion cell can be written as

$$
\begin{aligned}
& u(\mathbf{r})=u_{0}\left(\left(1 / 2 \pi R_{c}^{2}\right) \exp \left(-\left|\mathbf{A}_{c} \mathbf{r}\right|^{2} / 2 R_{c}^{2}\right)\right. \\
&\left.-\left((1-z) / 2 \pi R_{s}^{2}\right) \exp \left(-\left|\mathbf{A}_{s} \mathbf{r}\right|^{2} / 2 R_{s}^{2}\right)\right) .
\end{aligned}
$$

$u_{0}$ is a normalization factor such that $\int|u(\mathbf{r})|^{2} \mathrm{~d} \mathbf{r}=1$. The latter condition is equivalent to the restriction $\Sigma_{a} w_{a}^{2}=$ const enforced by Oja's and Yuille's update rules (Linsker 1990a) but assures in addition $G_{a a}=1 \forall a$ (see (8)). The matrices

$\mathbf{A}_{i}=\left(\begin{array}{rr}e \cos \left(\varphi_{i}\right) & e \sin \left(\varphi_{i}\right) \\ (-1 / e) \sin \left(\varphi_{i}\right) & (1 / e) \cos \left(\varphi_{i}\right)\end{array}\right), \quad i=c, s$

increase the absolute values of the exponents in (9) for $\mathbf{r} \|\left(\cos \left(\varphi_{i}\right), \sin \left(\varphi_{i}\right)\right)$ by $e^{2}$ and reduce them for the orthogonal direction. Evaluating $|\mathbf{A r}|^{2}$ and separating the terms into a part with rotational symmetry and a term with finite excentricity yields the equivalence

$$
\begin{aligned}
\exp \left(-|\mathbf{A r}|^{2} / 2 R^{2}\right)=\exp ( & \left.-r^{2} / 2 \widetilde{R}^{2}\right) \\
& \times \exp \left(-D(\mathbf{n} \cdot \mathbf{r})^{2} / 2 \widetilde{R}^{2}\right),
\end{aligned}
$$

where the dot $\cdot$ denotes the euclidean scalar product. The new parameters are the polarity $D=e^{4}-1$, the unit vector $\mathbf{n}=\mathbf{D} / D=(\cos (\varphi), \sin (\varphi))$ determining the direction of the polarity and the effective radius $\tilde{R}=e R$ with $\tilde{R} \simeq(1+D / 4) R$ for small polarities $D \ll 1$. Now each receptive field profile $u_{a}(\mathbf{r})$ is determined by a set of parameters $M_{a}=\left(R_{c a}, R_{s a}, \mathbf{D}_{c a}, \mathbf{D}_{s a}, z_{a}\right)$, where $\mathbf{D}=D \mathbf{n}$ is the polarity vector.

With (8) and (9), one can, in principle, calculate $G_{a b}$ for two arbitrary ganglion cells $a$ and $b$. However, this calculation is very time consuming and quickly becomes tedious. Therefore, (11) is simplified using the fact, that for $D \ll 1$ the first term on the right hand side of (11) attains large values only for small exponents $D(\mathbf{n} \cdot \mathbf{r})^{2}$ $2 \widetilde{R}^{2} \ll 1$ of the second term. So (11) can be approximated by expanding the latter exponential into a power series (Fig. 2d). Dropping all but the first two nonvanishing terms leads to

$\exp \left(-|\mathbf{A r}|^{2} / 2 R^{2}\right) \simeq \exp \left(-r^{2} / 2 \tilde{R}^{2}\right)\left(1-D(\mathbf{n} \cdot \mathbf{r})^{2} / 2 \tilde{R}^{2}\right)$.

Now it is straightforward to calculate the correlation matrix elements $G_{a b}$. Replacing $u_{a}$ and $u_{b}$ in (8) by (9), using the approximation (12) and neglecting all terms of order $O\left(D^{2}\right)$ leads to the following expression:

$$
\begin{aligned}
G_{a b}= & G\left(M_{a}, M_{b}, \mathbf{r}_{a b}\right) \\
= & u_{0, a} u_{0, b}\left(I_{c a, c b}\left(\mathbf{r}_{a b}\right)-\left(1-z_{b}\right) I_{c a, s b}\left(\mathbf{r}_{a b}\right)\right. \\
& \left.-\left(1-z_{a}\right) I_{s a, c b}\left(\mathbf{r}_{a b}\right)+\left(1-z_{a}\right)\left(1-z_{b}\right) I_{s a, s b}\left(\mathbf{r}_{a b}\right)\right),
\end{aligned}
$$

where

$$
\begin{aligned}
I_{1,2}(\mathbf{r})= & \left(1 / 4 \pi \tilde{R}_{12}^{2}\right) \exp \left(-r^{2} / 4 \tilde{R}_{12}^{2}\right) \\
& \times\left(K_{12}-\left(D_{1} R_{1}^{2}\left(\mathbf{n}_{1} \cdot \mathbf{r}\right)^{2}\right.\right. \\
& \left.\left.+D_{2} R_{2}^{2}\left(\mathbf{n}_{2} \cdot \mathbf{r}\right)^{2}\right) / 8 R_{12}^{4}\right)
\end{aligned}
$$


$K_{12}=1+\left(D_{1} R_{1}^{2}+D_{2} R_{2}^{2}\right) / 4 R_{12}^{2}$

$R_{12}=\left(1 / 2\left(R_{1}^{2}+R_{2}^{2}\right)\right)^{1 / 2}$

$\tilde{R}_{12}=\left(1 / 2\left(\left(1+D_{1} / 2\right) R_{1}^{2}+\left(1+D_{2} / 2\right) R_{2}^{2}\right)\right)^{1 / 2}$

and $1 \cong c a, s a, 2 \bumpeq c b, s b$. The correlation function $G\left(M_{a}, M_{b}, \mathbf{r}_{a b}\right)$ in (13) consists of four excentric gaussians each describing correlations due to the overlaps between one receptive field part (center or surround) of ganglion cell $a$ and one of ganglion cell $b$. At first sight the function seems to be translationally invariant, since only the difference vector $\mathbf{r}_{a b}$ appears. But $G_{a b}$ also depends on the two parameter sets $M_{a}$ and $M_{b}$ which may be different for each pair of ganglion cells. Therefore, only if all receptive field profiles have the same parameter values, the correlation function has translational invariance. If this is the case and if the ganglion cells are located on a square grid $\left(\mathbf{r}_{a}=(m, n), m, n=\right.$ $0, \pm 1, \ldots)$, the model is referred to as deterministic. In contrast, if there is some stochastic variation of the positions $\mathbf{r}_{a}$ and/or the parameters $\left\{M_{a}\right\}$, it is called a stochastic system.

\subsection{Characterization of the emerging receptive field structures}

Now measures for a quantitative description of the mature receptive field structure of the cortical cell are defined. The quantities considered are the orientation preference factor $l_{0}$ and the direction $\varphi_{0}$ of the orientation preference. Further measures are the mean spatial frequency $k_{0}$, the spatial frequency bandwidth $\Delta k$ and the orientational bandwidth $\Delta \varphi$. as

The receptive field profile of the cortical cell is given

$q(\mathbf{r})=\sum_{a} w_{a} u_{a}\left(\mathbf{r}-\mathbf{r}_{a}\right)$.

The squared maximal response of this cell to a sine wave grating with spatial frequency vector $\mathbf{k}$ can then be written as

$Q(\mathbf{k})=Q_{0}\left|(1 / 2 \pi) \int q(\mathbf{r}) \exp (-i \mathbf{k} \cdot \mathbf{r}) \mathrm{dr}\right|^{2} \equiv Q(k, \varphi)$,

which is independent of the phase of the receptive field as well as the phase of the input. $Q_{0}$ is a normalization factor such that $\int Q(\mathbf{k}) \mathrm{d} \mathbf{k}=1, k$ and $\varphi$ are the polar coordinates of $\mathbf{k}$. Because $\mathbf{k}$ and $-\mathbf{k}$ describe the same wave up to a phase, $Q(k, \varphi)=Q(k, \varphi+\pi)$ is valid. If the receptive field exhibits some orientation preference along $\varphi_{0}$, large values of $Q\left(k, \varphi_{0}\right)$ will result for appropriate $k$ (Fig. $3 \mathrm{a}, \mathrm{b})$. It is then convenient to define

$$
\begin{aligned}
& l_{0}\left(\cos \left(2 \varphi_{0}\right), \sin \left(2 \varphi_{0}\right)\right) \\
& =\int k \mathrm{~d} k \int \mathrm{d} \varphi(\cos (2 \varphi), \sin (2 \varphi)) Q(k, \varphi),
\end{aligned}
$$

which can be considered as a sum of vectors where those belonging to large $Q$ values point approximately into direction $2 \varphi_{0}$ and those with $\varphi \simeq \varphi_{0} \pm \pi / 2$ are more weakly weighted and point into the opposition direction (Fig. 3c). Hence the direction of the resulting vectors defines the direction $\varphi_{0}$ of the orientation preference, its length $l_{0}$ measures the average difference $Q(k, \varphi)-Q(k, \varphi \pm \pi / 2)$ for $\varphi \simeq \varphi_{0}$. Therefore, $l_{0}$ is called the orientation preference factor. It can be shown, that $l_{0}=0$ is obtained for symmetric receptive fields, $l_{0}=1$ for a pure sine wave as receptive field and $0 \leqslant l_{0} \leqslant 1$ for intermediate cases.

A further measure of orientation preference is the orientational bandwidth $\Delta \varphi$, which is given by

$\Delta \varphi^{2}=2 \int k \mathrm{~d} k \int_{\varphi_{0}-\pi / 2}^{\varphi_{0}+\pi / 2} \mathrm{~d} \varphi\left(\varphi-\varphi_{0}\right)^{2} Q(k, \varphi)$.

The average spatial frequency $k_{0}$ and the frequency bandwidth $\Delta k$ of the receptive field are

$k_{0}=\int k \mathrm{~d} k \int \mathrm{d} \varphi k Q(k, \varphi)$

$\Delta k^{2}=\int k \mathrm{~d} k \int \mathrm{d} \varphi k^{2} Q(k, \varphi)-k_{0}^{2}$.

Using these measures, a quantitative description of the emerging receptive field profiles can be given. This is of particular importance for the investigation of the stochastic model, where ensemble averages over these quantities must be taken to obtain statistically significant receptive field properties.

\section{The deterministic model: results}

In the deterministic model, all ganglion cell receptive fields are determined by the same set of parameter values ( $M_{a}=M \forall a$ ). Consequently the correlation matrix elements $G_{a b}$ depend only on the distance vector $\mathbf{r}_{a b}$ (translational invariance). This type of models has been investigated already by several authors (Linsker 1986, 1990a; Kammen and Yuille 1988; Yuille et al. 1989). Further the receptive field centers are located at the nodes of a square grid of width 1 . All length parameters $\left(R_{c}, R_{s}, R_{0}, \varrho\right)$ are given as multiples of this distance. Simulations were made using $\varrho=6,8,10$ and

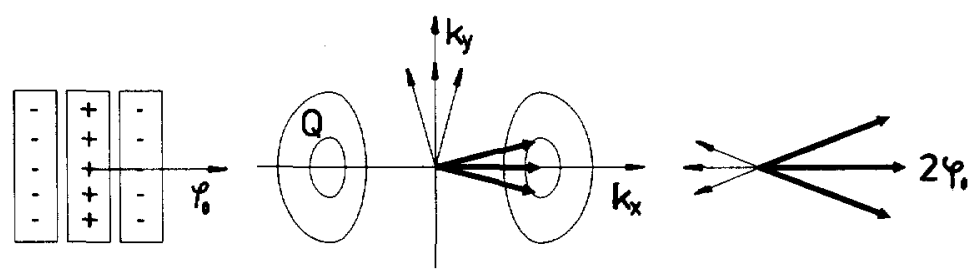

a b
Fig. 3. a A trilobed receptive field and a convenient definition of the orientation preference angle $\varphi_{0}$. b Contour plot of a hypothetical Fourier transform $Q(k, \varphi)$ of the receptive field in a. In equation (20) the thick vectors pointing approximately into direction $\varphi_{0}$ are more strongly weighted than the approximately orthogonal ones (thin vectors). $\mathbf{c}$ These two groups of vectors are subtracted from each other in (20), the resulting vector points into direction $2 \varphi_{0}$ 
12 which leads to systems with $N=113,197,317$ and 441 ganglion cells arranged within a circle of radius $\varrho$.

In this model network the largest eigenvalues and corresponding eigenvectors of the correlation matrix were calculated for each parameter set $M$. From the final vector $w$ the resulting receptive field profile was computed using (18) and characterized by the evaluation of $(20-23)$.

\subsection{The $R_{c}-R_{s}$ parameter space}

For the investigation of this parameter space balanced spatial antagonism and circular symmetry of the ganglion cell receptive fields is assumed, i.e. $z=D_{c}=D_{s}=0$.

As a first step, the largest eigenvalues and corresponding eigenvectors were calculated for $\varrho=10$. The receptive field center radius $R_{c}$ was varied, but its shape was kept fixed by setting $R_{s} / R_{c}=2=$ const. It was found, that all emerging receptive field profiles exhibit the sectored shape given by Linsker (1990a). However, using the notation defined in (1), only $(n, 0),(n, 1)$ and $(n, 2)$ type receptive fields emerged, which are drawn schematically for $n=0$ in Fig. 4a (in the present notation, which is slightly different from Linsker's (1990a), $n$ gives the number of nodes of the radial function). It turns out, that the $(n, 0)$ cells exhibit circular symmetry and the $(n, 1)$ cells have $2 n$-lobed orientation selective receptive fields. The widths of the lobes as well as the rings were found to be near the total receptive field center diameter $2 R_{0}$ of the ganglion cells (for the definition of $R_{0}$ see Fig. $2 \mathrm{c}$ ).

The dependence of the first five eigenvalues on $R_{c}$ is displayed in Fig. 4b. A linear term was subtracted from all eigenvalues to achieve a better resolution of their behaviour relative to each other. Comparison of the receptive field profiles with the maximal curve at this parameter value showed, that each curve belongs to receptive fields having the same number $l$ of angular nodes. For $l=1$ the eigenvalues are twofold degenerate and belong to an orthogonal pair of receptive fields, for $l=2$ the twofold-degeneracy is lifted due to the symmetry reduction brought into the system by the regularly located ganglion cells.

Figure $4 \mathrm{~b}$ and the behaviour of the orientation preference factor $l_{0}$ in Fig. $4 \mathrm{c}$ show, that there exist parameter domains where different $(n, l)$ types develop. Their boundaries can be evaluated by determining the crossing points of the eigenvalue curves in Fig. 4b.

To determine the shape of the domain boundaries in $R_{c}-R_{s}$ space, the simulations described above were repeated for different values of the ratio $R_{s} / R_{c}$ and for values of the projection radius $\varrho=6,8,10$ and 12 . The crossing points of the eigenvalue curves were then determined. One obtains the following results:

1. The domain boundaries for fixed $R_{s} R_{c}$ only depend on the relative magnitude $R_{0} / \varrho$ of the receptive field center, i.e. the absolute magnitude $\varrho$ of the system does not strongly influence the system behaviour. On the other hand it can be concluded, that the continuum limit often used to investigate similar models is a good
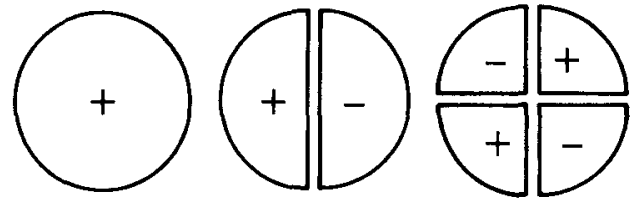

$(0,0)$

$(0,1)$

$(0,2)$
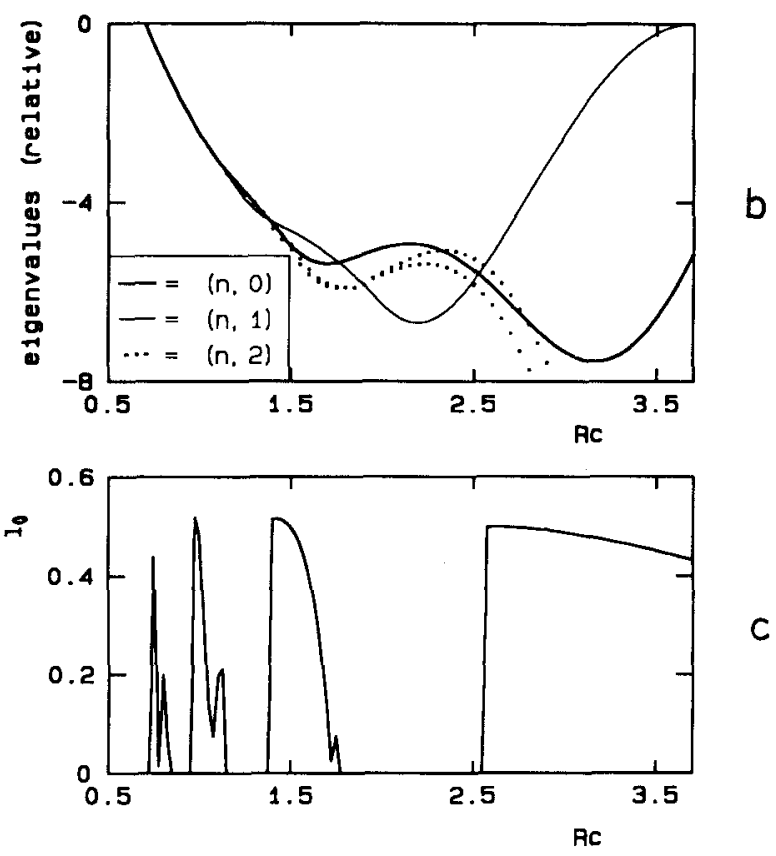

Fig. 4. a Schematic plot of the signs of the emerging synaptic connections $w_{a}$ for a $(0,0), \mathbf{a}(0,1)$ and a $(0,2)$ type receptive field. b Dependence of the largest five eigenvalues on the size of the receptive fields of the ganglion cells, where a linear function is subtracted from the eigenvalues. The $(n, 1)$-curve is twofold degenerate. The largest eigenvalue determines the type of the emerging receptive field. $c$ The behaviour of the orientation preference factor $l_{0}$ as a function of the radius $R_{c}$

approximation for even coarsely grained systems, as long as the receptive field centers of the ganglion cells are regularly spaced. An exception are the $(n, 2)$ domains which were found to disappear for $\varrho=12$ and which were not robust against changes of other parameters (see Sect. 3.3).

2. The parameter boundaries are nearly independent of $R_{s} / R_{c}$, only the relative receptive field size $R_{0} / \varrho$ determines the receptive field type emerging. This shows, that for fixed $R_{0}$ the shape of the receptive field surround of the ganglion cell does not strongly influence the results, provided $D_{c}=D_{s}=0$ holds.

3 . With decreasing $R_{0} / \varrho$, the relative splitting of the largest eigenvalues decreases strongly. Therefore, in this regime a nearly degenerate, multidimensional eigenspace exists, where all eigenvalues are very similar to the maximal one, so that the extraction of the principal component may require long simulation runs. Hence, any vector of constant length within this nearly degenerate subspace is a metastable solution during the maturation process of the receptive field. In general linear 
combinations of these solutions will lead to receptive field profiles consisting of excitatory and inhibitory patches. In particular, the dips of $l_{0}$ within the $(n, 1)$ domains for higher $n$ are due to a deformation of the receptive field profiles at regions where nearly degenerate eigenvalues occur and the numerical algorithm cannot compute Linsker's (1990a) true eigenvectors. It may be suggested that training mechanisms in real biological networks will fail, too.

Some of these results can be seen in Fig. 5a, where the parameter domains in the $R_{s} / R_{c}-R_{0} / \varrho$ space are displayed. Note, that only in $(n, 1)$ domains orientation selective receptive field profiles can form (though not by necessity, due to the twofold degeneracy), while in $(n, 0)$ domains, the emerging receptive field profile will always have circular symmetry.

The results obtained fit well to those given by Linsker (1986b), whose parameters $r_{F}$ and $r_{G}$ can be identified with $R_{0}$ and $\varrho$ respectively. With increasing $r_{G} / r_{F}$ Linsker described the emergence of trilobed, sixlobed and finally patchy receptive field profiles. These correspond to a horizontal walk through Fig. 5a. It is emphasized, that Linsker uses a different update rule, connection density and sometimes randomly distributed ganglion cell locations. The similarity of the behaviour of the two models therefore indicates strong robustness of the receptive field properties.

In the following, due to the near independence of the results of $\varrho$ and $R_{s} / R_{c}, \varrho=10$ and $R_{s} / R_{c}=2$ are chosen for the subsequent simulations.
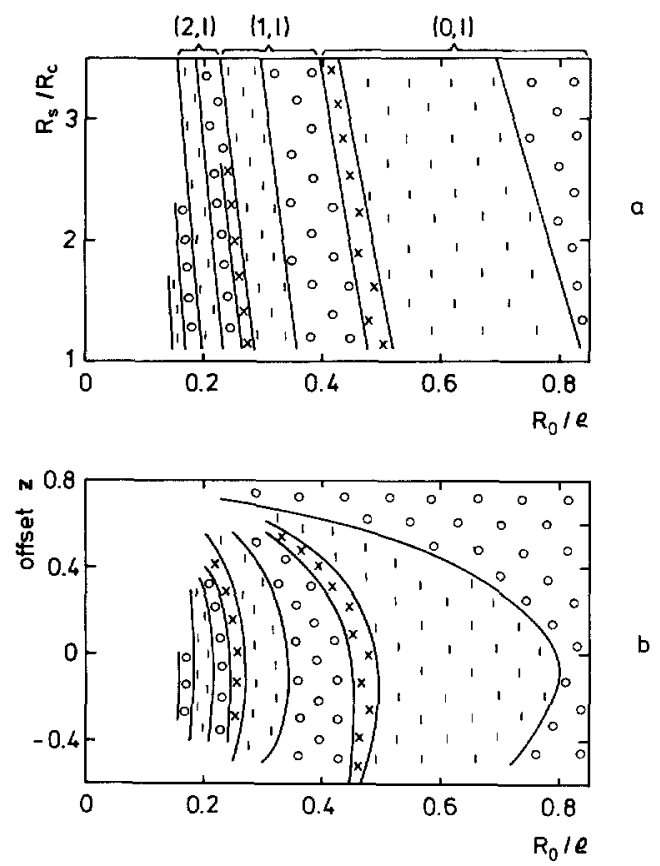

Fig. 5a,b. The parameter domains for the development of $(n, 0)$ (circles), $(n, 1)$ (bars) and $(n, 2)$ (crosses) type receptive fields. a The dependence of the parameter domain boundaries on the relative magnitude $R_{0} / \varrho$ of the receptive field centers of the ganglion cells and the ratio of the center and surround radii $R_{s} / R_{c}$. b The boundaries for varying offset $z$ of the receptive fields of the ganglion cells

\subsection{The influence of a nonvanishing offset $z$}

For $z \neq 0$ the receptive field profiles obtained are also sectored and can be characterized as $(n, l)$ type receptive fields. The boundaries separating parameter domains of these different receptive field types were determined as before for $z=-0.6 \cdots 0.7$, the result is displayed in Fig. $5 b$.

It can be seen, that for small offsets the boundaries become nearly independent of $z$. A strong antagonism $(z \leqslant-0.5)$ causes the last term in (13) to dominate the correlation matrix elements which then become positive. Therefore, in this regime all-excitatory or all-inhibitory receptive field profiles emerge (except for a small antagonistic surround due to the application of (18)).

In case of a weak antagonism of the receptive fields of the ganglion cells $(z \geqslant 0.3)$, all boundaries shift to smaller values of $R_{0} / \varrho$ and for $z \geqslant 0.7$ only the $(0,0)$ solution is obtained. In addition, the relative splittings of the largest eigenvalues in all but the $(0,0)$ domain decrease drastically incompassing a loss of robustness of the corresponding solutions. This behaviour is due to decreasing anticorrelations (terms 2 and 3 in (8)) which cause clusters of opposite response to emerge in the receptive field of the cortical cell.

Again, these results agree well with those obtained by Linsker (1986b), who observed the emergence of orientation selectivity while increasing the strength of spatial antagonism within the input layer. This corresponds to a vertical walk through the plot in Fig. 5b. The behaviour described above is also observed by Kammen and Yuille (1988) who showed that increasing spatial antagonism of the input neurons can lead to orientation selective receptive fields and that this behaviour can be understood as a symmetry breaking process.

\subsection{Eccentric ganglion cell receptive fields}

In the following results obtained in simulations with nonzero polarities $D_{c}, D_{s} \neq 0$ and $z=0$ i.e. orientation biased ganglion cell receptive fields with balanced antagonism $z$ are presented. First, only the receptive field center or the surround was allowed to deviate from circular symmetry, i.e. $D_{c} \neq 0, D_{s}=0$ or vice versa. The results obtained are:

1. Even for very small polarities $(D=0.1 ; e=0.025)$, the developing receptive field profiles are strongly orientation selective in all domains. They are no longer of $(n, l)$ type but rather consist of $2 n$ stripe shaped lobes in former $(n, 1)$ domains and of $2 n+1$ lobes in former $(n, 0)$ domains.

2. In $(n, 0)$ domains, the receptive field profiles show an increasing excentricity with increasing polarity $D$ and quickly reach their $2 n+1$ lobed appearance. In $(n, 1)$ domains, first the degeneracy is lifted and the receptive field, whose orientation preference angle is closest to the angle of polarity, corresponds to the maximum eigenvalue. With growing polarity $D$, this receptive field profile is then deformed to its final striped shape.

3. Finite center polarity $D_{c} \neq 0$ leads to stripe orientation along the prolonged axis $\left(\varphi_{0} \| \varphi_{c}\right)$, polarity of the 
surround leads to $\varphi_{0} \perp \varphi_{s}$. This is due to the fact, that the center predominantly causes positive correlation (center center interaction) while the surround predominantly causes anticorrelation (center surround interaction) of longer range for directions along the prolonged axis.

This behaviour agrees very well with the analysis of Yuille et al. (1989), who obtained these results for infinite projection radius $R_{0} / \varrho \rightarrow 0$. In addition it is shown, that their results are robust against the introduction of finite $\varrho$ and that there exist well defined domains, in which receptive field profiles of odd and even symmetry emerge. Receptive fields of intermediate phases were not observed.

Further, the behaviour of the model network for $D_{c}=D_{s} \neq 0, \varphi_{c}=\varphi_{s}$ was investigated. Here the orientation selectivity is less pronounced and in $(n, 0)$ domains also receptive fields with nearly circular symmetry appear. This is due to a competition between the center and surround polarities in this configuration. The weak orientation preference observed in $(n, 0)$ domains is not obtained in the analysis given by Yuille et al. (1989), because it is due to the boundary conditions which have been introduced using finite $\varrho$.

Finally, for rather small polarities $D<0.1$ the $(n, 2)$ domains disappear in the sense, that the $l=2$ eigenvalue never becomes the maximal one within this regime. This is another indication of the lack of robustness of this receptive field type.

In closing this section it should be mentioned, that orientation biased receptive fields have been described in biological systems by Levick and Thibos (1980) in case of retinal ganglion cells and by Shou and Leventhal (1989) for the LGN. The evidence from physiology suggests, that retinal ganglion cells prefer orientations that are parallel to the line connecting their receptive fields with the area centralis (fovea). In this arrangement neighboring cells have similar orientation bias, as has been assumed in our simulation discussed above. Although orientation bias has also been found in the relay cells of the LGN, the representation in the latter seems to be, at least qualitatively, similar to that found in the retina. Thus it seems justified not to assign any function to the LGN to a first approximation.

\section{The stochastic model: results}

In the following results obtained by applying static noise to the model network are discussed. As a starting point for the construction of a stochastic network, a deterministic system characterized by the ganglion cell positions $\mathbf{r}_{a}$ and the parameter set $M$ was taken. Scatter in the positions $\mathbf{r}_{a}$ was introduced by adding small vectors $\delta \mathbf{r}_{a}$ randomly chosen according to a twodimensional gaussian distribution of width $\Delta r$. To produce stochastic variations of the parameters, a set of random deviations $\delta M_{a}=\left(\delta R_{c a}, \delta R_{s a}, \delta \mathbf{D}_{c a}, \delta \mathbf{D}_{s a}, \delta z_{a}\right)$ was added to the deterministic set $M$ for each ganglion cell. The absolute values of the parameter deviations were randomly chosen according to gaussian distributions of widths $\Delta M=\left(\Delta R_{c}, \Delta R_{s}, \Delta D_{c}, \Delta D_{s}, \Delta z\right)$, the polarity angles $\varphi$ were uniformly distributed with probability $p(\varphi)=1 / \pi$. Hence:

$$
\begin{aligned}
M_{a}=M+\delta M_{a} & =\left(R_{c}+\delta R_{c a}, R_{s}+\delta R_{s a}, \mathbf{D}_{c}\right. \\
& \left.+\delta \mathbf{D}_{c a}, \mathbf{D}_{s}+\delta \mathbf{D}_{s a}, z+\delta z_{a}\right) \quad \forall a .
\end{aligned}
$$

The stochastic network is then determined by $M, \Delta M$ and $\Delta r$. The behaviour of this system can be characterized by the ensemble average of receptive field observables (for instance those defined in Sect. 2.4) over many system representations, each defined through $\left\{\mathbf{r}_{a}, M_{a} \mid a=1 \cdots N\right\}$.

Note, that the introduction of noise other than positional scatter violates the translational invariance of the correlation function of the system, so that the results obtained by Linsker (1990a), Kammen and Yuille (1988) and Yuille et al. (1989) are no longer valid for stochastic networks. Further, the continuum limits used in these investigations do not describe random positional fluctuations (which would require noisy connection densities) and therefore cannot model positional fluctuations.

\subsection{Noise of a single type}

Different types of static noise were applied separately to the system and changes occurring in the receptive field profiles were investigated using $\varrho=8$. For each of the parameter domains $(0,1),(0,2),(1,0),(1,1),(1,2)$ and $(2,1)$, only one representative parameter set $M$ was used with $R_{s} / R_{c}=2$ and $D_{c}=D_{s}=z=0$. Noise was added by continuously increasing a single width parameter from zero as starting point and taking a number of random samples (typically 20-50) for each deviation. Typical noise amplitudes applied were $\Delta R_{c} \leqslant 0.2 R_{c}, \Delta R_{s} \leqslant 0.2 R_{s}, \Delta r, \Delta D_{c}, \Delta D_{s}, \Delta z \leqslant 0.2$.

For positional fluctuations, offset fluctuations and fluctuations of $R_{c}$ and $R_{s}$ the qualitative behaviour of the model networks is similar.

1. The $(0,1)$ domain receptive field (bilobed) is robust against all parameter fluctuations within the limits given above. Its characteristics remain nearly constant. In addition, the twofold degeneracy is lifted, so that bilobed receptive fields will always form in a noisy system within the $(0,1)$ regime. This large robustness of the bilobed cells is an important finding as it parallels results from biological systems. In fact, bilobed receptive fields are the most frequent type of simple cell receptive fields found both in cat and monkey visual cortex (see introduction).

2 . With increasing noise, the $(1,0)$ domain receptive fields are continuously deformed to elliptic and trilobed shapes, i.e. $l_{0}$ steadily increases from $l_{0}=0$.

3 . In the $(n, 2)$ domains, even for very small noise amplitudes the receptive field profiles are strongly deformed and resemble $(n, 1)$ or deformed $(n, 0)$ profiles. This again gives rise to a weak robustness of these domains.

4. For small $R_{0} / \varrho$ values, the receptive field profiles deform to conglomerates of excitatory and inhibitory patches with increasing noise amplitudes. 
5. In $(n, 1)$ domains with $n>0$, the deformation processes generally lead to a significant decrease of the orientation preference, provided the noise amplitude is not too small.

The phenomenon of patch formation can be understood, if one considers the initial training rate for very small weights: $\Delta w_{a} \simeq \Sigma_{b} G_{a b} w_{b}$. If in a cluster of ganglion cells the receptive fields are either more densely packed, have weaker spatial antagonism or exhibit larger centers than the surrounding ones, the neurons in this cluster will have more (or stronger) positively correlated neighbour cells than anticorrelated ones. Therefore, if there is any initial offset of the weights $w_{b}$ in this area (for instance due to fluctuations or if they all have the same small initial value), all training rates $\Delta w_{a}$ of this cluster, which are dominated by the $G_{a b}>0$ terms in the sum above, will have the same sign and a patch of uniformly signed weights emerges. In contrast to the maturation process in a deterministic system, a cluster emerging through $G_{a b}$ fluctuations has a fixed location which is determined by the correlation matrix and not by initial fluctuations in the synaptic couplings $w_{b}$. Clusters of this type prevent the system from arriving at the eigenstates of the deterministic model.

The network is found to behave qualitatively different, when random polarities are applied. For all domains except $(0,1)$, which does not change very much, the orientation preference factor $l_{0}$ increases with increasing $\Delta D_{c}$ or $\Delta D_{s}$. This amplification of orientation preference is even more pronounced, if $\Delta D_{c}$ and $\Delta D_{s}$ are switched on simultaneously.

The angle $\varphi_{0}$ of orientation preference is found to be correlated with the angle $\varphi_{d}$ of the resulting polarity vector $\mathbf{D}=(1 / \mathrm{N}) \Sigma_{a} \mathbf{D}_{a}$. For $\Delta D_{c} \neq 0, \Delta D_{s}=0, \varphi_{0}$ tends to be oriented in the same direction as the resulting polarity while for $\Delta D_{c}=0, \Delta D_{s} \neq 0$ the preferred orientation corresponds to $\varphi_{d} \pm \pi / 2$. This agrees with the results obtained for the deterministic model which becomes more transparent, if one considers the average polarity to cause orientation preference along or orthogonal to its direction and the remaining zero average random polarities to make this result noisy.

\subsection{Strong and combined noise}

In a second step, large amplitude single type static noise and combined noise of several parameters was applied to the model. Then one finds two qualitatively different types of behaviour corresponding to the large $R_{0} / \varrho$ regime and the small $R_{0} / \varrho$ regime. But there is no fixed boundary between these regimes rather a continuous change of behaviour is observed. In the $(0,1),(0,2)$ and $(1,0)$ domains, no qualitatively new behaviour can be seen, i.e. the $(0,1)$ type is robust, the $(0,2)$ type unstable and the $(1,0)$ type becomes deformed to a roughly trilobed orientation selective shape.

In contrast, for small values of $R_{0} / \varrho$ a new kind of maturation behaviour becomes dominant (while for weak noise, only a tendency to exhibit this behaviour is visible). Large amplitude noise causes a significant part of the mature efficacies to vanish almost, hence, the corresponding synapses are nearly cut off and will be neglected therefore. The remaining nonzero synapses form a receptive field with a radius smaller than the projection radius $\varrho$. The field is mostly deformed but never consists of separate patches of nonzero synapses. The effect of size reduction of the receptive fields was found to be significant. This may be seen from an evaluation of the behaviour of the spatial frequency bandwidth $\Delta k$, which increases when the receptive field size and the number of lobes is reduced.

Further, if several types of noise are applied simultaneously to the system, this effect is even more pronounced. The resulting receptive fields typically have a diameter $R \simeq 6 R_{0}$. Without random polarities, receptive fields with large orientation preference $\left(l_{0}>0.4\right)$ as well as nearly circular symmetric ones emerge. If random polarities are added, the receptive fields are mostly arranged as orientation selective profiles of three up to five lobes. A four-lobed receptive field which formed in a system with combined noise is displayed in Fig. 6a, a receptive field with approximately circular symmetry is shown in Fig. 6 b.

Two principles guiding the receptive field maturation process suggest an explanation for this decoupling phenomenon. These are the variation of training rates due to local static fluctuations of the correlations and the competition of the synaptic efficacies caused by the nonlocal, norm restricting, property of the learning rule (for instance $\Sigma_{b} w_{b}^{2}=$ const. for Oja's and Yuille's rules). Clusters of neurons which are correlated to many other cells or are more strongly correlated to others (due to static noise), have large initial training rates. The efficacies of the neurons in these clusters will grow faster than the remaining ones. They finally take the main part of the restricted norm $\|w\|$ and force all other efficacies to remain small. This corresponds to an evolutionary principle, where the cluster of cells with the largest Hebbian training rates represents the fittest population of those competing for the norm of the weight vector.

As a consequence, only the cluster with the largest initial training rates will survive in a mature system and will have large synaptic efficacies. The efficacies of the surrounding ganglion cells will become smaller with rising distance from this cluster due to the decreasing correlations with the cluster cells. This explains, why no separate receptive field patches are observed. Further, the larger the initial training rates of a cluster are, the larger its efficacies finally become and the more surrounding synapses become cut off. Therefore combined noise, which produces the largest fluctuations, leads to the smallest receptive field profiles.

To test this suggestion, the mature state of a deterministic system was calculated. In a cluster of five ganglion cells $R_{c}$ and $R_{s}$ were enlarged by a factor 1.2 , so that cells in the cluster had more correlated neighbour cells than neurons far from the cluster. The result of this simulation is shown in Fig. 6c. In fact, only the cells of the cluster and those correlated with them reach large efficacies, the remaining cells become decoupled. The training rates of the cluster cells are larger in an 


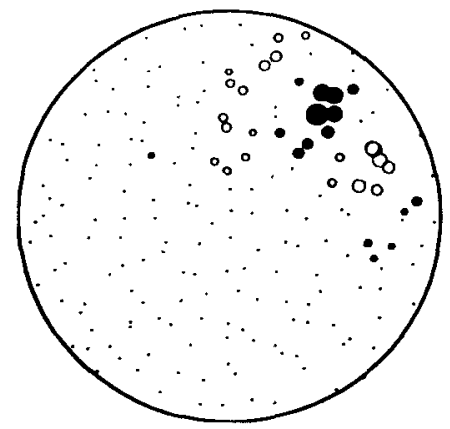

a

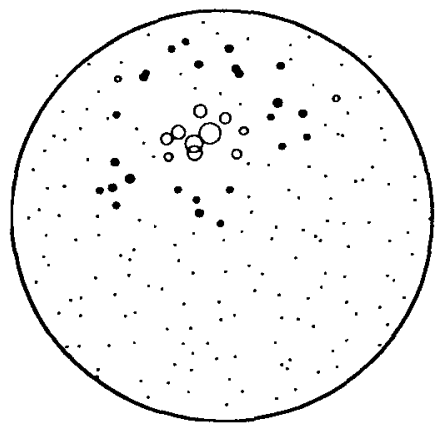

$\mathrm{b}$

Fig. 6a-c. Results of simulations for stochastic model networks with $\varrho=8, R_{c}=0.8, R_{s}=1.6, D_{c}=D_{s}=z=0$. Filled circles denote positive, open circles negative synaptic effcacies. The area of the circles is proportional to the synaptic strength. Simulations a and $\mathbf{b}$ used $\Delta r=0.3, \Delta R_{c}=0.15, \Delta R_{s}=0.3, \Delta D_{c}=\Delta D_{s}=0.2$ and $\Delta z=0.3$ as

intermediate state of the maturation process, because there are more contributing terms in $\Sigma_{b} G_{a b} w_{b}$ than for distant cells. Hence, these synaptic weights grow quickly and afterwards make large contributions to the training rates of the cells correlated with them. As a result a small $(2,0)$ type receptive field with a diameter $R \simeq 6 R_{0}$ emerges. This behaviour is robust against the presence of noise as long as it produces static fluctuations which do not exceed the artificial one. If so, the fluctuation with the largest amplitude matures as a receptive field. These results of a deterministic network thus strongly support the explanations given above for a stochastic network.

\section{Discussion}

The main purpose of this work was to test the robustness of results obtained using a deterministic model network against the introduction of several kinds of static noise. It was found, that the behaviour of the deterministic system is highly sensitive to the presence of noise. While the $(0,1)$ type receptive fields do not change very much, all other types become strongly deformed, when static noise is applied. In particular, they can no longer be classified by the symbols $(n, l)$ and no characteristic parameter domains can be separated. Because of this lack of robustness, a stochastic system seems necessary when modeling the emergence of receptive field profiles in biological neural networks, which themselves are noise systems.

The investigation of stochastic systems showed a feature which cannot be found in the deterministic model. For not too large ratios $R_{0} / \varrho$ it was found, that the size of the emerging receptive field profiles is mainly determined by the average radius $R_{0}$ of the receptive field centers of the ganglion cells and not by the projection radius $\varrho$. This effect is due to static noise and also due to a nonlocal rule limiting the norm of the synaptic vector. Possible biological implementations of such a rule could be the competition of the synapses for the

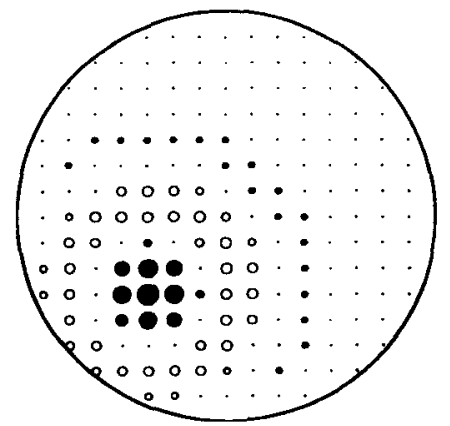

$\mathrm{C}$

noise amplitudes, the orientation preference factors obtained are $l_{0}=0.43$ for a and $l_{0}=0.17$ for $\mathbf{b}$. c Resulting synaptic distribution for a deterministic system, where the receptive fields of the five ganglion cells with the largest positive efficacies were enlarged by a factor 1.2

membrane surface or for ion channels within the membrane of the postsynaptic neuron. Hence, there may be some biological relevance to the mechanism of receptive field size reduction through synapse decoupling mentioned above. This property of the stochastic system leads to some interesting consequences which are discussed below.

If one considers neighbouring cortical cells in layer IVc, these cells will have approximately similar projection radii, because this quantity can be understood as an average over many arborization radii of the afferent ganglion cell (or LGN cell) axons. As a consequence the deterministic model would predict, that the receptive fields of all cortical cells have approximately the same size, the same number of lobes or rings (depending on $R_{0} / \varrho$ ) and would overlap each other nearly completely.

This does not, however, correspond to the situation found in biological systems. In fact, the receptive field sizes vary considerably and they are randomly located (scattered) within an aggregate field with more than twice the average receptive field diameter (Hubel and Wiesel 1974; Schiller et al. 1976a). Further, only bilobed or trilobed profiles are found and the widths of the lobes vary with receptive field diameter. Finally, if one assumes $\underline{Q}_{0} \geqslant 0.5 \mathrm{~mm}$ for immature systems (which is reasonable, since mature afferents mostly project to two or more ocular dominance stripes which are separated by about $0.8-1 \mathrm{~mm}$ (Lund 1988; see also Humphrey et al. 1985 for kittens)) the emerging receptive fields of layer IVc cells are smaller than these values of $\varrho$ would predict. For instance, at one degree of visual angle from the fovea, the magnification factor $m$ is found to be $4 \mathrm{~mm} /$ degree, so that $\varrho_{0}=0.5 \mathrm{~mm}$ would lead to a receptive field size of $(1 / 4)^{\circ} \times(1 / 4)^{\circ}$, which is just the projection area $2 \varrho / m \times 2 \varrho / m$ (Hubel and Wiesel 1974). These authors found the receptive field profiles to have this average size, but they averaged over all cortical laminae. In fact, layer IVc cells have much smaller receptive fields than all other cortical cells. Hence, they are smaller than the average receptive 
field size and therefore must be considerably smaller than the projection area.

These receptive field properties emerge in a natural way, if one models the maturation process for each cell out of a small patch of layer IVc using a stochastic neural network. In this respect, a short quantitative description of the connectivity situation in macaque monkey layer IVc is given, but one should emphasize that this attempt has to be taken as a rather coarse estimate.

The total area of macaque monkey area 17 is $1300 \mathrm{~mm}^{2}$. It receives input from about $1.5 \times 10^{6}$ afferent fibres from each LGN and therefore from each retina (Hubel and Wiesel 1977). Each $\mathrm{mm}^{2}$ of cortical layer IVc tissue will contain afferents from roughly 1200 ganglion cells each having roughly 3000 synaptic connections to cortical cells. Further, from the neuron density of about $10^{5} / \mathrm{mm}^{3}$ for monkeys (Rockel et al. 1980), one can estimate layer IVc to contain about $2 \cdot 10^{4}$ cells per $\mathrm{mm}^{2}$, which then receive $4 \cdot 10^{6}$ afferent terminals. This leads to the situation, that each of those layer IVc cells receives input from about 200-300 ganglion cells and that, for $\varrho_{0}=0.5 \mathrm{~mm}$, on average only every fifth cortical neuron is connected to one particular ganglion cell. Therefore, a cluster of cortical cells can be modeled by stochastic network systems (one for each cortical cell) with $\varrho=8$ or $\varrho=10$ projection areas (leading to about 200-300 afferents), which overlap nearly completely for all cells. Each of these systems has an individual set of input neurons (ganglion cells) which is different from those of the other cells.

For each cortical cell the widths of the lobes of their receptive fields is determined by the maximum radius $R_{0}^{\max }$ of the receptive field centers of the ganglion cells. For not too large values of $R_{0}^{\max }$ (for instance $R_{0}^{\max }=2$ ), the diameter of the emerging receptive field is roughly $6 R_{0}^{\max }$ which leads to profiles with three up to five lobes located anywhere within the projection area. Since different cells receive input from different sets of ganglion cells, after maturation each cell will possess another receptive field size, but all diameters will be smaller than $2 \varrho / \mathrm{m}$. Further, all receptive fields will consist of only few lobes, the widths of which also vary for different cortical cells. The receptive fields will be randomly located within the projection area (scatter). In particular, for $R_{0}^{\max } \simeq 1.5, \varrho=8$ and $R_{0} \simeq 2, \varrho=10$, the receptive field diameter is about one half of the projection diameter and the scatter will be of the same order of magnitude, which was in fact seen by Hubel and Wiesel (1974) for the monkey. Finally, the emerging receptive field profiles will exhibit a continuum of orientation preference strengths from nearly circular symmetric to strongly orientation selective receptive fields. Both cell types are found in layer IVc by Hubel and Wiesel (1968) and Schiller et al. (1976a). This shows, that for biologically relevant parameter sets, the predictions obtained using the stochastic model agree very well with physiological data found in biological systems. While our model emphasizes the simulation of early maturation of receptive fields before structured visual input becomes available, some short- comings of the current model must be mentioned also. First of all, many of the neglected features of biological systems like time dependent behaviour, nonlinear neuronal responses, the role of the LGN and lateral interactions, which we are currently investigating, may be of importance for modelling self organization processes of receptive fields of cortical cells. For instance, cells with orientation tuning curves as narrow as $9^{\circ}$, which were found by Schiller et al. (1976b), did not form in our model network. This extreme narrowing might be due to lateral inhibition within layer IVc, where neighbouring cells suppress the activity of the considered cell when the stimulus orientation slightly deviates from the optimal orientation of his cell. This is corroborated by Wörgötter and Koch (1991), who discussed the role of lateral inhibition for the response behaviour of mature neural networks. These authors showed, that different inhibitory mechanisms lead to a significant narrowing of orientation tuning curves obtained from their model neurons. The contributions of differently weighted short- and long-range lateral inhibition lead to biologically relevant orientation tuning without destroying other response characteristics of the model network. It is thus obvious, that without the introduction of lateral inhibition between cortical cells sufficiently narrow orientation tuning curves as observed in biological systems (Schiller et al. 1976) could not be obtained with the present single cell model.

Further, the receptive field profiles obtained neither match very closely the profiles found by Hubel and Wiesel (1962), nor do they resemble Gabor functions as found by Daugman (1985). Instead, more or less deformed receptive fields with strongly differing strengths of their orientation preference were found.

For these cells, it is not straightforward to simulate the formation of orientation columns with currently discussed model networks. This is partly because orientation preference is not very sharply defined within a set of noisy receptive fields, partly because most of the present models concerned with feature map formation are deterministic ones. The modeling of feature map foundation in noisy network systems will thus be a challenging new topic for future investigations, we are currently performing.

\section{Summary}

The self organization of the receptive field of a simple cell was modeled using a deterministic model neural network with Hebb type update rule and a stochastic model, which was obtained from the deterministic one by adding different types of static noise to the receptive fields of the input neurons. The deterministic model was found to include the results obtained by several authors using similar models. Their results naturally arose within the concept of parameter domains for $(n, 0)$ and $(n, 1)$ cells, into which the parameter space of the system is divided. The introduction of polar receptive fields of ganglion cells produces strongly orientation selective cortical cells similar to those predicted for 
$R_{0} / \varrho=0$ by Yuille et al. (1989). Further, the increase of spatial antagonism crosses the parameter domains in a way with produces the results found by Linsker (1986b) and described as a symmetry breaking process by Kammen and Yuille (1988).

However, most of these results were found to be highly sensitive to the presence of static noise in the model neural network. The concept of parameter domains is no longer useful in noisy systems. Instead one observes a new kind of maturation characteristic, where many synaptic connections are cut off and which is in fact assumed to occur in maturing cortical tissue. The receptive field profiles emerging within this stochastic system closely resemble experimental data about size, scatter and structure of the receptive fields of layer IVc cortical simple cells. Therefore, static noise has turned out to be a crucial ingredient to the simulation of self organization processes in biological neural networks and future work should investigate all consequences of the presence of noise on the behaviour of the deterministic model neural networks considered so far.

\section{References}

Brown TH, Kairiss EW, Keenan CL (1990) Hebbian synapses: Biophysical mechanisms and algorithms. Annu Rev Neurosci 13:475-511

Daughman JG (1985) Uncertainty relation for resolution in space, spatial frequency, and orientation optimized by two-dimensional visual cortical filters. J Opt Soc Am A 2:1160-1169

Hebb DO (1949) The organization of behaviour. Wiley, New York

Hubel DH, Wiesel TN (1959) Receptive fields of single neurones in the cat's striate cortex. J Physiol (London) 148:574-591

Hubel DH, Wiesel TN (1962) Receptive fields, binocular interaction and functional architecture in the cat's visual cortex. J Physiol (London) 160:106-154

Hubel DH, Wiesel TN (1968) Receptive fields and functional architecture of monkey striate cortex. J Physiol (London) 195:215243

Hubel DH, Wiesel TN (1974) Uniformity of monkey striate cortex: A parallel relationship between field size, scatter, and magnification factor. J Comp Neurol 158:295-306

Hubel DH, Wiesel TN (1977) Functional architecture of macaque monkey visual cortex. Proc R Soc London Ser B 198:1-59

Humphrey AL, Sur M, Uhlrich DJ, Sherman SM (1985) Projection patterns of individual $\mathrm{X}$ - and $\mathrm{Y}$-cell axons from the lateral geniculate nucleus to cortical area 17 in the cat. J Comp Neurol 233:159-189

Kammen DM, Yuille AL (1988) Spontaneous symmetry-breaking energy functions and the emergence of orientation selective cortical cells. Biol Cybern 59:23-31

Levick WR, Thibos LN (1980) Orientation bias of cat retinal ganglion cells. Nature 286:389-390

Linsker R (1986a) From basic network principles to neural architecture: Emergence of spatial-opponent cells. Proc Natl Acad Sci USA 83:7508-7512

Linsker R (1986b) From basic network principles to neural architecture: Emergence of orientation-selective cells. Proc Natl Acad Sci USA 83:8390-8394

Linsker R (1990a) Designing a sensory processing system: What can be learned from principal component analysis. In: Proceedings of the International Joint Conference on Neural Networks (IJCNN), (Washington, DC, 16-19 January 1990)

Linsker R (1990b) Perceptual neural organization: Some approaches based on network models and information theory. Annu Rev Neurosci 13:257-281

Lund JS (1988) Anatomical organization of macaque monkey striate visual cortex. Annu Rev Neurosci 11:253-288

Marčelja S (1980) Mathematical description of the responses of simple cortical cells. J Opt Soc Am 70:1297-1300

Mumford D (1991) On the computational architecture of the neocortex. I. The role of the thalamo-cortical loop. Biol Cybern $65: 135-145$

Oja E (1982) A simplified neuron model as a principal component analyzer. J Math Biol 15:267-273

Rockel AJ, Hiorns RW, Powell TPS (1980) The basic uniformity in structure of the neocortex. Brain 103:221-244

Schiller PH, Finlay BL, Volman SF (1976a) Quantitative studies of single-cell properties in monkey striate cortex. I. Spatiotemporal organization of receptive fields. J Neurophysiol 39:1288-1319

Schiller PH, Finlay BL, Volman SF (1976b) Quantitative studies of single-cell properties in monkey striate cortex. II. Orientation specificity and ocular dominance. J Neurophysiol 39:1320 -1333

Shou T, Leventhal, AG (1989) Organized arrangement of orientation-sensitive relay cells in the cat's dorsal lateral geniculate nucleus. J Neurosci 9:4287-4302

Wiesel TN, Hubel DH (1974) Ordered arrangement of orientation columns in monkeys lacking visual experience. J Comp Neurol 158:307-318

Wörgötter F, Koch C (1991) A detailed model of the primary visual pathway in the cat: Comparison of afferent excitatory and intracortical inhibitory connection schemes for orientation selectivity. J Neurosci 11:1959-1979

Yuille AL, Kammen DM, Cohen DS (1989) Quadrature and the development of orientation selective cortical cells by Hebb rules. Biol Cybern 61:183-194 